\title{
Apuntes sobre el cálculo de umbrales de notificación en el control de concentraciones empresariales $^{(*)}$

\section{Enrique Felices SaAvedra}

Abogado por la Pontificia Universidad Católica del Perú. Master of Laws - LL.M._- por la Universidad de Yale. Profesor del curso de Control de Concentraciones en la Pontificia

Universidad Católica del Perú.

Ex Secretario Técnico de la Comisión de Fiscalización de la Competencia Desleal del Instituto Nacional de Defensa de la Competencia y de la Protección de la Propiedad Intelectual.

Rodrigo López VilLanueva

Abogado por la Pontificia Universidad Católica del Perú. Adjunto de docencia del curso de Control de Concentraciones en la

Pontificia Universidad Católica del Perú. Ex funcionario de la Comisión de Defensa de la Libre Competencia del Instituto Nacional de Defensa de la Competencia y de la Protección de la Propiedad Intelectual.

SUMARIO:

I. Introducción.

II. La definición de umbrales de notificación.

III. ¿Qué umbrales y por qué?

IV. Algunas reglas especiales para el cálculo de umbrales.

1. Fondos de inversión.

2. Empresas estatales.

3. Comercio exterior.

V. Nota final. 


\title{
RESUMEN:
}

En este artículo los autores analizan los umbrales de notificación en el control de concentraciones empresariales desde una perspectiva conceptual y práctica atendiendo a su desarrollo en la doctrina, la legislación comparada y las guías orientativas de distintos países e instituciones para así presentar una visión completa y didáctica sobre la materia.

Palabras clave: control de concentraciones, competencia, umbrales de notificación, concentración empresarial.

\begin{abstract}
:
In this paper, the authors analyze the notification thresholds in merger control based on a conceptual and practical perspective. This, considering its treatment in doctrine, comparative law and guidelines from different countries and institutions in order to present a complete and grounded vision on the subject.

Keywords: merger control, competition law, notification thresholds, mergers.
\end{abstract}

\section{INTRODUCCIÓN}

A pesar de que nuestro país cuenta con un marco legal y una política de competencia desde hace más de 30 años, es uno de los pocos de ingresos medios o altos que no contaba con un régimen general de control de concentraciones empresariales.' Es cierto que desde el año 1997, con la publicación de la Ley 26876, Ley Antimonopolio y Antioligopolio del Sector Eléctrico, se incorporó el control previo a las operaciones de concentración en el sector eléctrico, pero tanto en su aplicación como en su alcance, se trató de una experiencia bastante limitada.

La reciente promulgación de la Ley 31112, ley que establece el control previo de operaciones de concentración empresarial, y su reglamento, aprobado mediante Decreto Supremo № 0392021-PCM —en adelante, el "Reglamento de la Ley $31112 "-2$, nos confrontan con un escenario muy distinto, que presentará desafíos tanto a la autoridad como a las empresas que desarrollan actividades económicas en el mercado.

Uno de los elementos centrales para la aplicación de este nuevo marco legal es el de los umbrales de notificación. En este trabajo abordaremos los umbrales desde una perspectiva conceptual y también práctica, atendiendo a su desarrollo en la doctrina, en la legislación comparada y en las guías orientativas de distintos países e instituciones, con el fin de presentar una visión lo más completa y didáctica posible sobre esta materia y contribuir a una discusión pública que apenas empieza.

\section{LA DEFINICIÓN DE UMBRALES DE NOTIFI- CACIÓN}

Las razones que conducen a las empresas a concentrarse son diversas, pero en general se originan en su interés por generar una mejor rentabilidad, ya sea obteniendo una mayor

1. En este sentido, ver el documento Exámenes Inter-Pares de la OCDE y el BID del derecho y política de competencia: Perú" (Perú: OCDE, 2018) 135:

Más de cien países prevén en sus legislaciones de competencia regímenes de control de concentraciones \entre ellos todos los países que forman parte de la OCDE, salvo Luxemburgo, que en todo caso está sujeto al régimen de control de concentraciones de la UE. Todos esos países reconocen que problemas de competencia de naturaleza estructural pueden ser consecuencia de determinadas operaciones de concentración, y que un mecanismo de control de concentraciones puede contribuir a evitar que consoliden estructuras de mercado susceptibles de tener efectos anticompetitivos significativos, difícilmente contrarrestables por otros medios.

2. El presente trabajo ha sido preparado con anterioridad a la entrada en vigencia de la Ley 31112 y su reglamento, aprobado mediante Decreto Supremo №. 039-2021-PCM. 
participación de mercado, desarrollando nuevas actividades para reducir su exposición al riesgo, ampliando su portafolio de productos, buscando propiciar sinergias con empresas que complementan una oferta de servicios, persiguiendo una posición de liderazgo en un mercado, u optimizando sus procesos tecnológicos, de producción o de distribución. En este sentido, las operaciones de concentración son consustanciales al funcionamiento de la economía e indispensables para imprimir dinamismo en los mercados, permitir que las empresas crezcan y prosperen, y que se genere bienestar para los consumidores y la sociedad en su conjunto.

En algunas circunstancias, sin embargo, esto no es así y esas operaciones se convierten en materia de preocupación para las instituciones que velan por la competencia en el mercado. En efecto, ciertas operaciones de concentración pueden resultar en la creación o el fortalecimiento del poder mercado con que cuentan ciertas empresas y posibilitar la afectación de la dinámica competitiva, restringiendo o eliminando la competencia efectiva y, con ella, afectando el bienestar de los consumidores. ${ }^{3}$ Esto es particularmente cierto en mercados con estructuras oligopólicas, ${ }^{4}$ en los que la concentración del poder de mercado puede disipar con mayor facilidad las presiones competitivas que propician, precisamente, ese dinamismo competitivo que se traduce en bienestar para los consumidores. $^{5}$

Frente a esta realidad, las legislaciones de la mayor parte de los países de ingresos medios y altos cuentan con un régimen general de control de concentraciones, cuyo propósito es el de identificar y evaluar, antes de que sean consumadas, aquellas operaciones que estén en capacidad de ocasionar un daño a la competencia o de producir, en los términos utilizados por la Ley 31112, ", "una restricción significativa de la competencia en los mercados involucrados."

En este sentido, el documento de Exámenes Inter-Pares de la Organización para la Cooperación y el Desarrollo Económico - $\mathrm{OCDE}$, y el BID del Derecho y Política de Competencia, Perú 2018, explica que "Ia aplicación de sistemas de notificación previa se justifica en que es mucho más sencillo prevenir un problema de restricción de la competencia derivado de una operación con consecuencias estructurales que solucionar ese problema ex post", pues, añade, "permite frenar operaciones restrictivas de la competencia susceptibles de irrogar perjuicios a los consumidores al coartar la libre competencia entre empresas rivales y/o eliminar competidores".?

3. Al respecto, el Artículo. 1 de la Ley 31112 señala lo siguiente: "La presente ley tiene por objeto establecer un régimen de control previo de operaciones de concentración empresarial con la finalidad de promover la competencia efectiva y la eficiencia económica en los mercados para el bienestar de los consumidores."

4. A mayor abundamiento, ver el Artículo 25 del Reglamento 139/2004 — Reglamento comunitario de concentraciones:

"Habida cuenta de las consecuencias que las concentraciones pueden tener en estructuras de mercado oligopolísticas, resulta particularmente necesario preservar la competencia efectiva en estos mercados. Si bien muchos mercados oligopolísticos presentan un elevado grado de competencia, en determinadas circunstancias las concentraciones que implican la desaparición de importantes presiones competitivas que las partes en la concentración ejercían entre sí, así como una reducción de la presión competitiva sobre los competidores restantes, pueden, incluso en ausencia de la probabilidad de coordinación entre los miembros del oligopolio, llegar a ser un obstáculo significativo para la competencia (...)".

5. En el mismo sentido, Herbert, Hovernkamp y Carl, Shapiro, "Horizontal Mergers, Market Structure, and Burdens of Proof", The Yale Journal, (2018): Acceso el 25 de febrero de 2021 https://scholarship.law.upenn.edu/cgi/viewcontent.cgi?Artículoicle=2935\&context=faculty_scholarship

6. Ver Artículo 7 de la Ley 31112.

7. OCDE, Exámenes Inter-Pares de la OCDE y el BID del derecho y política de competencia: Perú (Perú: OCDE, 2018) 136 
$Y$, sin embargo, determinar a priori y con certeza qué operaciones tienen la capacidad de ocasionar "restricciones significativas de la competencia" es una tarea compleja, por no decir imposible. En primer lugar, porque más allá de la técnica aplicada, el análisis de operaciones de concentración es por definición prospectivo y, por lo mismo, subjetivo e incierto. En segundo lugar, porque someter todas y cada una de las concentraciones que se lleven a cabo al análisis de una autoridad de competencia sería impracticable, además de ineficiente: el costo de hacerlo sería exorbitante para la autoridad y para los agentes involucrados $y$, con seguridad, sería un ejercicio fútil e improductivo, en la medida en que un gran porcentaje de dichas operaciones no tienen la posibilidad real de generar una restricción significativa de la competencia.

Por esta razón, las legislaciones de competencia son unánimes al establecer mecanismos que permitan segmentar las operaciones de concentración que se llevan a cabo en el mercado, de forma tal que solo estén sujetas a su revisión y análisis las que presentan, según ciertos criterios, una mayor probabilidad de causar un perjuicio a la competencia.

\section{III. ¿QUÉ UMBRALES Y POR QUÉ?}

La principal manera en que las distintas legislaciones sobre control de concentraciones seg- mentan las operaciones que se realizan en los mercados es a través del establecimiento de umbrales de materialidad. De esa forma, solo aquellas operaciones que superan los umbrales deben ser notificadas ante la autoridad. ${ }^{8}$ Esto presupone, naturalmente, que nos encontremos frente a una operación que califique previamente como un acto de concentración y que, además, tenga un nexo geográfico con el país. ${ }^{9}$

De un lado, solo aquellos actos u operaciones que impliquen "una transferencia o cambio de control de una empresa o parte de ella"10 calificarán como un acto de concentración y, por lo tanto, deberán ser notificados ante la autoridad, entendiéndose que dicho cambio de control debe tener un carácter duradero -es decir, no ser temporal o incidental-y permitir a quien lo adquiere la posibilidad de ejercer una influencia decisiva y continua sobre una empresa. ${ }^{11}$

A dichos efectos, se entiende que existe un control permanente cuando el acto de concentración tiene la potencialidad de modificar la estructura del mercado y, con ello, la interacción competitiva existente. ${ }^{12}$ Si bien las transferencias de propiedad o acuerdos sin límite temporal suelen representar un cambio de control permanente, un acuerdo con duración definida también puede configurarse como tal si es que el tiempo de vigencia es lo suficientemente largo como para modificar la estructura del mercado. ${ }^{13}$

8. Sin perjuicio de esta regla general, cabe tener en cuenta que, en el Perú, el Artículo 6.4 de la Ley 31112 ha previsto también un régimen de notificación voluntaria para aquellos actos de concentración que no superen los umbrales, así como la posibilidad de que la autoridad de competencia pueda revisar de oficio operaciones de concentración que no los hayan superado.

9. OECD, Local Nexus and Jurisdictional Thresholds in Merger Control (2016), 4 y 5.

10. Ver Articulo 5 de la Ley 31112.

11. Ver definición de "Control" en el Artículo. 3 de la Ley 31112 y, complementariamente, entre muchos otros, el numeral 3 del Artículo 3 del Reglamento 139/2004 - Reglamento comunitario de concentraciones) o el Artículo. 47 del Decreto Ley 211 de Chile y la Guía Práctica para la aplicación de umbrales de notificación de operaciones de concentración en Chile de la Fiscalía Nacional Económica.

12. Ver página 8 de la exposición de motivos del Reglamento de la Ley 31112.

13. Ver numeral 28 de la "Comunicación consolidada de la Comisión Europea sobre cuestiones jurisdiccionales en materia de competencia, realizada de conformidad con el Reglamento (CE) No 139/2004 del Consejo, sobre el control de las concentraciones entre empresa". 
Sobre la base de lo anterior, a nivel de la Unión Europea, se ha considerado que existe un cambio de control permanente cuando el acto de concentración tiene una duración de entre ocho - 8- y quince - 15- años. ${ }^{14}$ No obstante, cabe tener en cuenta que dichos plazos no reflejan criterios rígidos, sino que, como ha señalado la agencia de competencia chilena, corresponde a un análisis de caso por caso, en el que se tomará también en cuenta, entre otros, "las características propias de la transacción, el mercado en que tiene lugar, o los ciclos de la actividad económica." 15

Dicho esto, ¿qué es un acto de concentración? En general, es todo aquello que produzca un cambio de control como consecuencia de una fusión entre dos o más empresas o partes; la adquisición por una o varias partes, de manera directa o indirecta, de derechos que le confieran control sobre una o varias entidades; la constitución de una empresa en común; y la adquisición de control sobre un activo productivo. ${ }^{16}$ Por el contrario, no se consideran actos de concentración aquellos que son producto del crecimiento interno de un agente económico, o resultado de operaciones realizadas dentro de su grupo económico, así como tampoco aquellos en los que el cambio de control sea de naturaleza temporal, ya sea que responda a un mandato legal o contractual, o a una particularidad intrínseca a una actividad. ${ }^{17}$

Del otro, solo aquellas concentraciones que tengan un nexo geográfico o vínculo suficiente con el país serán notificables. Es decir, no bastará que una operación involucre a una empresa local para que deba ser notificada en el país, sino que la operación en cuestión debe estar en capacidad de tener "efectos competitivos apreciables" en él.

Y aunque existe consenso en la legislación comparada sobre la necesidad de establecer mecanismos para evitar notificaciones innecesarias y el uso indebido de recursos para la revisión de concentraciones que no tengan la capacidad de ocasionar "restricciones significativas de la competencia", esos mecanismos específicos varían entre los países.

Quizá la necesidad de encontrar una metodología idónea para evitar intervenciones innecesariamente costosas de las autoridades de competencia haya sido mejor sintetizada hace más de 30 años por Frank H. Easterbrook en su trabajo The Limits of Antitrust..$^{18}$ En esa investigación, Easterbrook se refería al ensayo y error propios de la intervención gubernamental en la aplicación de las normas de competencia, ya sea a través de tribunales o autoridades administrativas, ${ }^{19}$ y a los costos que ese ejercicio impone sobre los agentes en el mercado. En ese marco, argumentaba que las políticas de competencia debían abandonar el objetivo de impedir todas y cada una de las conductas que pudieran tener un efecto anticompetitivo y, con ello, reducir los costos de los errores que propicia la consecución de esa tarea imposible. Con ese fin, el tra-

14. Al respecto, ver el numeral 18 de la "Comunicación consolidada de la Comisión Europea sobre cuestiones jurisdiccionales en materia de competencia, realizada de conformidad con el Reglamento (CE) No 139/2004 del Consejo, sobre el control de las concentraciones entre empresa".

15. Fiscalía Nacional Económica, Guía de Competencia (2017), 14

16. Ver Artículo. 5.1 de la Ley 31112.

17. Sobre este punto, revisar: International Competition Network, Recommended Practices for Merger Notification and Review Procedures, 2018, disponible en https://www.internationalcompetitionnetwork.org/wp-content/ uploads/2018/09/MWG_NPRecPractices2018.pdf

18. Frank H Easterbrook, The Limits of Antitrust, 63 TEX. L. REV. 1 (1984).

19. Easterbrook, The Limits of Antitrust, $p$ 4: "Antitrust is costly. The judges act with imperfect information about the effects of the practices at stake. The costs of action and information are the limits of antitrust". 
bajo de Easterbrook, apoyado en herramientas de la estadística, hacía hincapié en los costos causados por ciertas clases de errores en que incurren las autoridades: los "Errores Tipo I" o "Falsos Positivos", causados por decisiones que condenan ciertas conductas por considerarlas anticompetitivas cuando en realidad no lo son, y los "Errores Tipo II" o "Falsos Negativos", causados por decisiones que fallan al no detectar y condenar ciertas conductas que sí son anticompetitivas, en ambos casos con el consiguiente perjuicio a los consumidores y el mercado.

El análisis que realiza Easterbrook es igualmente relevante para el control de concentraciones, pues nos permite indagar en los criterios y consideraciones que entran en juego para la fijación de los umbrales de notificación: ¿Cuál es, después de todo, la mejor combinación posible para minimizar los errores que puede causar una autoridad de competencia frente a una operación de concentración?

Establecer umbrales muy altos, por ejemplo, tendría por efecto reducir el número de operaciones notificables. Al ser menores en número, se reducirían los costos administrativos en que incurre la autoridad para su revisión, así como los costos de transacción que la notificación impone sobre las partes. Únicamente desde una perspectiva de costos, establecer umbrales altos podría parecer la decisión correcta. Lo cierto, sin embargo, es que esa decisión podría generar a su vez un efecto perjudicial para el mercado si es que tiene como resultado que no se notifiquen y revisen aquellas operaciones que sí cuentan con la capacidad de causar "restricciones significativas de la competencia". En este sentido, fijar umbrales muy altos podría propiciar Errores Tipo II, al permitir que se lleven a cabo sin ser notificadas operaciones que cuentan con la capacidad de generar efectos anticompetitivos importantes.

De otro lado, establecer umbrales muy bajos sería a todas luces un ejercicio ineficiente, y podría propiciar Errores Tipo I, pues capturar, como en la pesca de arrastre, la mayor cantidad posible de transacciones y someterlas al escrutinio de la autoridad generaría enormes costos administrativos y de transacción, además de afectar la competencia y el bienestar de los consumidores si es que la injerencia de la autoridad se traduce en la prohibición de conductas que no son realmente anticompetitivas.

La realidad, sin embargo, es más compleja y la solución no se encuentra en uno u otro extremo, pues, como apunta Lambert, las conductas que se analizan bajos las reglas de la competencia tienen en la práctica efectos procompetitivos y anticompetitivos a la vez.. ${ }^{20}$ Quizá sea por ello que, aunque el International Competition Network -en adelante, "ICN"21 — o la OCDE ${ }^{22}$ inciden en la importancia de establecer umbrales claros, objetivos y predecibles, no existe un tratamiento uniforme en la legislación comparada sobre la combinación óptima de elementos para su establecimiento.

20. Thomas Andrew Lambert, The Limits of Antitrust in the 21st Century (2020). University of Missouri School of Law Legal Studies Research Paper Number 2020-06, disponible en: https://ssrn.com/abstract=3533549 or http://dx.doi. org/10.2139/ssrn.3533549

Traducción libre del siguiente texto: "These are typical of the behaviors antitrust addresses: They involve both upsides and downsides and thus may be, on net, either output-enhancing (procompetitive) or output-reducing (anticompetitive). They are, in short, mixed bags."

21. Ver: International Competition Network, "Recommended Practices for Merger Notification and Review Procedures" -2018), disponible en https://www.internationalcompetitionnetwork.org/wp-content/uploads/2018/09/ MWG_NPRecPractices2018.pdf 
De hecho, reconociendo la dificultad de proponer un sistema unívoco, el ICN recomienda una serie de criterios a nivel de políticas de competencia para la fijación de los umbrales. Ellos incluyen ${ }^{23}$, entre otros, los siguientes:

a) La identificación previa de los objetivos que se quiere alcanzar — por ejemplo, reducir el número de operaciones notificables por año, de tal forma que la capacidad de gestión de la autoridad no sea desbordada y que los recursos se destinen únicamente a aquellas pocas operaciones consideradas problemáticas; aunque el objetivo podría también ser inverso, es decir, incrementar el número de operaciones notificables para evitar Errores Tipo II-;

b) El tipo de umbral que se considera apropiado - por ejemplo, existen umbrales que se calculan sobre la base de ventas o ingresos obtenidos en el país, en contraposición con las ventas o ingresos globales de las empresas involucradas en la operación y en no pocos casos es posible ver una combinación de ambos;

c) La dispensa para operaciones que no están en capacidad de generar efectos anticompetitivos importantes - por ejemplo, transacciones que suponen la reorganización interna dentro de un mismo grupo empresas, o la adquisición de participaciones minoritarias que no transfieren control-;

d) La determinación del número de notifi- caciones que considera deseable recibir cada año - por ejemplo, en economías más pequeñas con autoridades de competencia que cuentan con alcances limitados-;

e) El tamaño de la economía del país - que condujo a Suecia, por ejemplo, a reducir aD sus umbrales, sustituyendo el criterio de ventas globales por aquellas obtenidas en el país por las empresas involucradas en la operación de concentración-;

f) La comparación con jurisdicciones similares - criterio que, por ejemplo y como veremos más adelante, fue utilizado para la determinación de los umbrales contenidos en la Ley 31112 y en el Decreto de Urgencia 013-2019 que la precedió, tomando como base el promedio de los umbrales de ventas individuales y conjuntas para la notificación en los países de la OCDE-; o

g) La posibilidad de revisar de oficio operaciones que no superaban los umbrales —facultad que, como en el caso peruano, suele estar aparejada de umbrales relativamente altos-.

Tomando estos criterios en cuenta, la mayor parte de las legislaciones de países que pertenecen a la OCDE - ver cuadro- establecen umbrales sobre la base del valor de ventas o del valor de activos, pues estos se consideran los más claros y objetivos. Y porque "permiten a las autoridades enfocarse en transacciones que involucran a entidades de cierta envergadura económica y con un vínculo local suficiente."24

22. OECD, Local Nexus and Jurisdictional Thresholds in Merger Control, (2016), https://www.oecd.org/officialdocuments/publicdisplaydocumentpdf/?cote=DAF/COMP/WP3-2016)4\&docLanguage $=$ En

23. Ver: International Competition Network, "Recommended Practices for Merger Notification and Review Procedures" (2018), https://www.internationalcompetitionnetwork.org/wp-content/uploads/2018/09/MWG_NPRecPractices2018.pdf p. 8

24. OECD, Local Nexus...p 6. 


\section{Criterios usados en los umbrales de la notificación previa ${ }^{25}$}

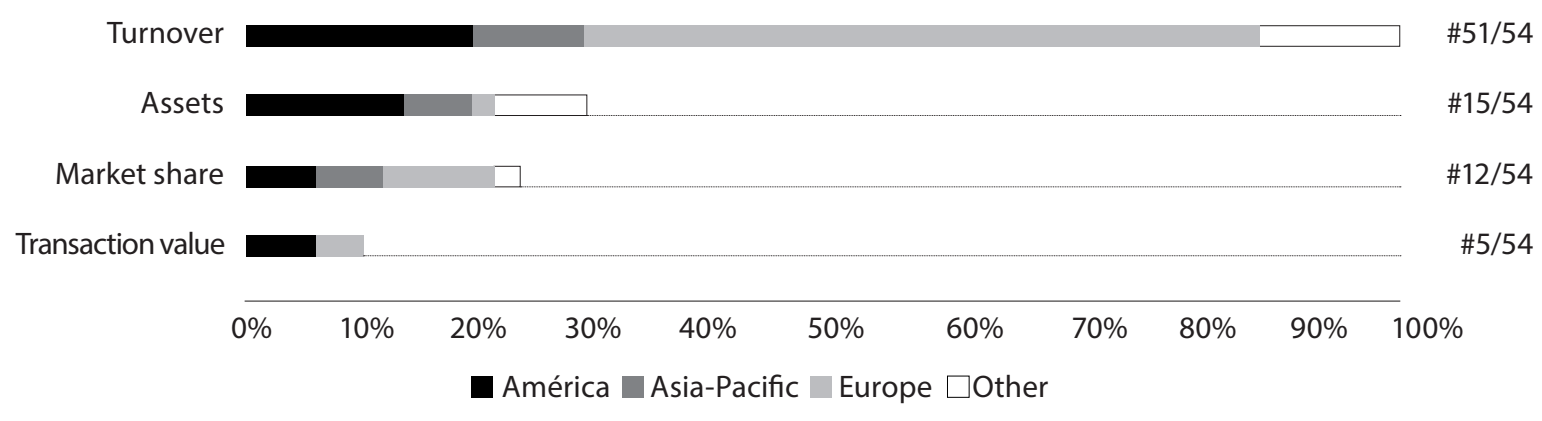

En la misma dirección apuntan las recomendaciones del ICN,${ }^{26}$ que remarcan la necesidad de que los umbrales sean claros, simples y fácilmente administrables, ${ }^{27}$ proponiendo que estos se fijen según criterios objetivamente cuantificables como el valor de los activos, o las ventas o ingresos de las empresas involucradas en la operación, dejando de lado criterios subjetivos o discrecionales. ${ }^{28}$

En este sentido, criterios como las ventas o los ingresos sirven para estimar los recursos eco- nómicos que se combinan mediante una operación de concentración, en lugar de enfocarse únicamente en las entidades jurídicas que formalmente llevan a cabo la transacción. Así, estas métricas operan como sucedáneos del poder de mercado para efectos de la notificación, e incluyen por lo general las ventas y los ingresos en el país del grupo económico del que las empresas involucradas formen parte. A diferencia de otras métricas, la información contable sobre ventas e ingresos es de muy simple acceso para las empresas involucradas y

25. OECD. Global merger control. OECD Competition Trends. Volumen II (2021) p 13.

26. Ver International Competition Network, "Recommended Practices for Merger Notification and Review Procedures" -2018), p.5, disponible en: https://www.internationalcompetitionnetwork.org/wp-content/uploads/2018/09/ MWG_NPRecPractices2018.pdf

27. En el mismo sentido, Thomas Servières señala:

"Los umbrales basados en el volumen de ventjas tienen la ventaja de aportar seguridad jurídica tanto a las autoridades de competencia como a los agentes económicos. Se introdujeron a nivel de la UE porque son fácilmente cuantificables, verificables y constituyen una buena aproximación para estimar la relevancia económica de las partes que se fusionan en un territorio determinado, así como el carácter transfronterizo de la transacción. En resumen, constituyen una manera conveniente para la Comisión de capturar a la mayoría de las fusiones potencialmente problemáticas que tienen un impacto dentro de la UE".

Traducción libre de: "Turnover-based thresholds have the advantage of bringing legal certainty for both competition law enforcers and economic entities. They were introduced at EU-level because they are easily quantifiable, verifiable and they constitute a good proxy for estimating the economic weight of the merging parties in a given territory as well as the cross-border character of this transaction. In sum, they constitute a convenient way for the Commission to capture most of the potentially problematic mergers having an impact within the EU." Thomas Servières, "'Value-of-transaction' threshold in EU merger control: filing an enforcement gap or overcomplicating already complex proceedings?", p. 46. En ICC Global Antitrust Review, 2017.

28. Ibíd, p. 5: "Así, a pesar de sus imperfecciones, se prefieren los umbrales basados en criterios objetivos: el reto está en establecer los criterios adecuados".

Traducción libre de: "Thus, despite their imperfections, thresholds based on objective criteria are preferred: the challenge is in establishing the appropriate criteria." 
para la autoridad, estando por lo demás reflejadas en sus balances o estados financieros.

No debe extrañarnos, por ello, que la Ley 31112 haya establecido umbrales sobre la base de dichos criterios. ${ }^{29}$ En efecto, el artículo 6.1 de la Ley 31112 establece que una operación de concentración empresarial estará sujeta al procedimiento de control previo cuando de manera concurrente se alcancen dos umbrales: (i) un umbral conjunto, según el cual la suma total del valor de las ventas o ingresos brutos anuales o valor de activos en el Perú de las empresas involucradas en la operación de concentración empresarial haya alcanzado durante el ejercicio fiscal anterior a aquel en que se notifique la operación, un valor igual o superior a 118,000 Unidades Impositivas Tributarias —en adelante, "UIT"-, y (ii) un umbral individual, según el cual el valor de las ventas o ingresos brutos anuales o valor de activos en el Perú de al menos dos de las empresas involucradas en la operación de concentración empresarial haya alcanzado, durante el ejercicio fiscal anterior a aquel en que se notifique la operación, un valor igual o superior a 18,000 UIT, cada una. ${ }^{30}$

En este sentido, la inclusión de los conceptos de ventas o ingresos en la Ley 31112 como criterios para determinar si una operación debe ser notificada es consistente con las recomendaciones de la OCDE y el ICN, pues además de ser criterios claros y objetivos, permiten a las autoridades enfocarse en aquellas transacciones que involucran a partes de cierta relevancia económica y con vínculo suficiente con el Perú. $Y$ en efecto, estos son los criterios utilizados en la fijación de umbrales en las tres cuartas partes de las economías de la OCDE. ${ }^{31}$

Aunque un número importante de las economías de la OCDE calculan sus ventas o ingresos en el país en el que produce la operación, un número significativo de países incluyen también en este cálculo a las ventas o ingresos de fuente mundial. Los Estados Unidos, Francia y Alemania son algunos de los países que utilizan criterios mixtos de notificación, siendo el umbral de ventas o ingresos de fuente mundial siempre mayor que el local. $Y$ en algunos pocos casos, el nexo geográfico se establece por criterios distintos a las ventas o ingresos, como el de los "efectos producidos por la operación", tal como ocurre en los Estados Unidos. Finalmente, ciertos países combinan las ventas y los ingresos con el valor de activos como lo establece la Ley 31112, por ejemplo-, o con el valor total de la transacción o con la participación de mercado de las partes, como veremos más adelante. ${ }^{32}$

A nivel regional cabe también tener en cuenta la Recomendación del Parlamento Andino 415 - Marco normativo para formular y armonizar políticas de regulación sobre el control previo de concentraciones y fusiones empresariales en los países de la región andina-, mediante

29. De hecho, estos umbrales ya estaban establecidos en el Decreto de Urgencia 013-2019, publicado el 13 de noviembre de 2019 en el diario oficial El Peruano y sustentados tanto en su Exposición de Motivos como en el Informe No 024-2019-ST-CLC-INDECOPI del 6 de marzo de 2019, que fue emitido por la Secretaría Técnica de la Comisión de Libre Competencia en el marco de la elaboración del Proyecto de Ley No 4110/2018-PE, que fue presentado por el Poder Ejecutivo al Congreso de la República mediante Oficio № 073-2019-PR del 26 de marzo de 2019.

30. Artículo 6.1 de la Ley 31112.

31. Según el Oficio № 257-2020-PR que envió la Presidencia de la República a la Presidencia del Congreso con observaciones a la Ley que establece el control previo de operaciones de concentración empresarial que fue aprobada por insistencia, el 76\% de economías de la OCDE utilizan el nivel de ventas o ingresos brutos como único criterio para fijar umbrales.

32. OECD, Local Nexus and Jurisdictional Thresholds in Merger Control, (2016). 
el cual se propone que los umbrales (i) tengan una naturaleza objetiva y monetaria y (ii) que estos consideren únicamente las ventas a nivel del país y no a nivel internacional. ${ }^{33}$

Ahora bien, una vez establecidos los criterios que serán utilizados para la determinación de los umbrales, corresponde definir cuál es el valor que debe asignarse a dichos umbrales. En el caso peruano, se consideró que la manera apropiada de hacerlo era mediante un análisis comparativo basado "en la revisión de los umbrales de notificación de otros países con experiencia en la aplicación de control de concentraciones. ${ }^{134}$ Y para ello se tomó como referencia a los países de la OCDE, pues al ser parte de ella han adoptado, presumiblemente, las mejores prácticas y estándares internacionales. Ese análisis comparativo concluyó que si se utilizaba "el valor promedio de los umbrales de ventas conjuntas e individuales de notificación de la lista de la OCDE como umbrales referenciales de partida, obtenemos un valor de 149 millones de dólares para el umbral de ventas conjuntas y de 22.5 millones de dólares para el umbral de ventas individuales, ${ }^{135}$ valores que expresados en soles y convertidos a UIT, corresponden a las
118,000 UIT y 18,000 UIT establecidas en la Ley $31112 .{ }^{36}$

No obstante ello, es relevante preguntarse hasta qué punto los valores así definidos son realmente precisos y económicamente adecuados. Después de todo, más allá de resultar de un análisis comparativo ente los países que forman parte de la OCDE, lo cierto es que el proceso de determinación de umbrales es en esencia el producto de una deliberación política y no solo técnica. Por ello, entre otras circunstancias, se recomienda que el valor que se establezca para los umbrales sea reevaluado periódicamente para confirmar o ajustar su idoneidad. Del mismo modo se pronuncian la OCDE y el ICN, y así se establece en el artículo 6.3 de la Ley 31112, pudiendo el Instituto Nacional de Defensa de la Competencia y de la Protección de la Propiedad Intelectual —en adelante "INDECOPI" - proponer la actualización del valor de los umbrales, siempre que se justifique su necesidad y se apruebe a través de una norma con rango de ley.

Es importante notar que el Decreto de Urgencia 013-2019 no incluía el valor de los activos

33. Al respecto, ver literales a) y b) del Artículo 14 del texto propuesto por la Recomendación 415 del Parlamento Andino:

"Artículo 14.- Determinar "Umbrales" monetarios como herramienta central.- Las nuevas tendencias para destacar operaciones de concentración y fusiones de empresas que pueden afectar la libre competencia o generar conductas anticompetitivas, más allá de indicadores de concentración referenciales, se basa en los denominados "Umbrales" (Límites Mínimos) de las transacciones realizadas. Por ello se propone se considere:

a) Establecer Umbrales, definidos como parámetros mínimos cuantificables a partir de los cuales se decidirá evaluar o no una operación de concentración y fusión de empresas, lo que deberán ser de tipo monetario, siendo recomendable, según la práctica internacional, aquellas relacionadas con el valor de las ventas, los ingresos de las empresas y el valor de la transacción, realizada entre las empresas involucradas.

b) Los umbrales deben ser selectivos en el ámbito geográfico, es decir, deben de considerar las ventas a nivel de país y no a nivel internacional."

34. Ver: Exposición de Motivos al Decreto de Urgencia que Establece el Control Previo de Operaciones de Concentración Empresarial, página 11 y el Informe № 024-2019-ST-CLC-INDECOPI.

35. Página 10 de la Exposición de Motivos del proyecto de ley de control de concentraciones presentado por la Presidencia de la República del Perú ante el Congreso.

36. Es importante notar que el texto sustitutorio aprobado en el pleno del Congreso de la República el 2 de mayo de 2019 no consideraba los mismos valores. De hecho, se había establecido el valor de 25,000 UIT para el calculo de los umbrales individuales, siendo ese valor luego reconsiderado al no ajustarse al análisis comparado con la OCDE ya mencionado. 
dentro de los criterios para la determinación de los umbrales, elemento que fue incorporado posteriormente en la Autógrafa que dio lugar a la Ley 31112. Y es interesante ver que, dentro de las observaciones presentadas en dicha ocasión, se argumentó que el valor de activos como criterio de notificación no era adecuado en tanto no era un buen sucedáneo del poder de mercado de los agentes y que por esta razón debería descartarse, pues, de un lado, no todos los activos con los que cuentan las empresas son productivos $-\mathrm{y}$, en consecuencia, no todos ellos inciden directamente en su posición competitiva-, y, del otro, porque no todos los activos están en la misma capacidad de generar retornos o ingresos homogéneos para las empresas. Por esta razón, se concluye, su inclusión incrementaría "innecesariamente la carga procesal del INDECOPI debilitando la eficiencia y eficacia del control de concentraciones empresariales. ${ }^{137}$

Coincidimos en que, desde la perspectiva de la autoridad y de las partes notificantes, la gestión de umbrales basados en valor de ventas o ingresos es más simple que una basada en el valor de los activos, además de ser con seguridad menos gravosa para las partes y menos costosa desde la perspectiva de la carga procesal para la administración. No obstante ello, tanto la OCDE como la ICN consideran que el valor de los activos es también un criterio apropiado, pues, al igual que las ventas o los ingresos, son objetivamente medibles en el curso habitual de sus actividades en el país, aunque en estos casos siempre sean aplicados en combinación con otros criterios - usualmente las ventas o ingresos de la empresa, pero también en ocasiones en con el valor total de la transacción o la participación de mercado de las empresas involucradas-. ${ }^{38}$

Claro está, como bien mencionaba Margrethe Vestager, Comisaria Europea de Competencia:

"El asunto es que no siempre son las ventas las que hacen que una empresa sea atractiva para una fusión. En ocasiones, lo que importa son los activos. Ellos pueden ser una base de clientes o incluso un conjunto de datos. En el sector farmacéutico, puede ser un nuevo medicamento que está en desarrollo, pero cuya venta aún no ha sido aprobada. O una compañía puede ser valiosa simplemente por su capacidad para innovar. Una fusión que involucra esta clase de compañías podría claramente afectar la competencia, incluso si las ventas de esa empresa no sean tan altas para alcanzar los umbrales. Así que, al mirar únicamente las ventas, podríamos estar perdiendo de vista algunas operaciones importantes que deberíamos revisar. ${ }^{139}$

Es interesante anotar que tanto la OCDE como el ICN reconocen que la participación de mercado de las empresas involucradas en una operación es, quizás, el mejor sucedáneo del poder de mercado para efectos de la notifica-

37. Ver página 2 del Oficio № 257-2020-PR, mediante el cual la Presidencia de la República presentó observaciones a la autógrafa de ley del Congreso.

38. No obstante los argumentos en contra reflejados en las observaciones antes mencionadas.

39. Margrethe. Vestager, Refining the EU Merger Control System (2016), disponible en —https://ec.europa.eu/commission/commissioners/2014-2019/vestager/announcements/refining-eu-merger-control-system_en). Traducción libre de:

"The issue seems to be that it's not always turnover that makes a company an attractive merger partner. Sometimes, what matters are its assets. That could be a customer base or even a set of data. In the pharmaceutical sector, it might be a new drug that's been developed but not yet approved for sale. Or a company might be valuable simply because of its ability to innovate. A merger that involves this sort of company could clearly affect competition, even though the company's turnover might not be high enough to meet our thresholds. So by looking only at turnover, we might be missing some important deals that we ought to review". 
ción y, por ello, el factor predictivo más exacto sobre la capacidad que tiene una operación para generar efectos anticompetitivos. ${ }^{40}$ Esta apreciación tiene sentido en la medida que la determinación de una participación de mercado presupone haber definido previamente los mercados de producto y geográfico sobre los que se calculan dichas participaciones $y$, al hacerlo, confirmar la existencia de un nexo geográfico suficiente con el país de que se trate. Y a pesar de su precisión, la participación de mercado como criterio de notificación es desaconsejada por ambas organizaciones porque su determinación es laboriosa e impone costos innecesarios sobre las partes, además de no ser de fácil acceso, como sí lo son los criterios de ventas o ingresos. Es por ello que, a pesar de ser utilizados en ciertas jurisdicciones, se emplean siempre en combinación con otros criterios.

Sin perjuicio de lo anterior, no hay que perder de vista que, incluso en el caso en que se cuente con información suficiente para cuantificar la cuota de mercado y la adquirente tenga una mayor a $50 \%$, esto no es suficiente para concluir que exista poder de mercado. Ello, en tanto que, como señala Craig W. Conrath, a fin de determinar la existencia de una posición de dominio se deben considerar varios elementos, entre ellos, las barreras de entrada, las características de producción y el exceso de capacidad productiva de las partes. ${ }^{41}$ Por ello que, incluso en el supuesto que se invirtieran recursos para determinar la cuota de mercado, esto por sí solo tampoco garantiza que se capturen operaciones que, necesariamente, vayan a generar un efecto adverso a la competencia.
Finalmente, debe mencionarse el valor total de la transacción como criterio de notificación. Este es quizás el más polémico $y$, al mismo tiempo, el más actual, pues es probablemente el que mejor se presta para enfrentar concentraciones en los mercados digitales.

Esto es relevante porque, siguiendo a Vestager, la economía digital no es simplemente un sector de entre otros que puede afectarnos como personas que compran o venden productos, sino que está en el centro de todo y puede cambiar no solo la manera en que vivimos, sino las oportunidades que tenemos. ${ }^{42}$

Quizás el ejemplo emblemático y el mejor caso de estudio sobre la limitación que muestran los umbrales convencionales para hacer frente a concentraciones en mercados digitales sea todavía la adquisición de Whatsapp por Facebook en el 2014. ${ }^{43}$

Como se sabe, Facebook pagó un monto mayor a los 19 mil millones de dólares por Whatsapp, una empresa que ya entonces tenía 600 millones de usuarios. Se trataba de una operación que afectaba a 1,700 millones de usuarios a nivel mundial y a más de 300 millones en Europa, con una participación de mercado combinada cercana al 40\%. Y, sin embargo, como los servicios ofrecidos por Whatsapp eran mayormente gratuitos y le reportaban ventas menores a los 20 millones de dólares, la operación no superaba los umbrales vigentes $y$, por lo tanto, no existía la obligación legal de notificarla ante la Comisión Europea. La operación fue finalmente notificada ante la Comisión, claro está, pero gracias al sistema de reenvíos que los estados

40. Ello sin perjuicio del análisis de fondo que debe realizar posteriormente la agencia de competencia, contrastando la participación de las partes con otros factores que permitan determinar si es que existe o no una posición de dominio; por ejemplo, la existencia de barreras considerables a la entrada y salida del mercado relevante. En el caso de la Ley 31112, estos criterios se encuentran recogidos en el numeral 2 del Artículo 7.

41. Craig Conrath, Practical Handbook of antimonopoly law enforcement for an economy in transition. Section 8-8.

42. Vestager, Refining the EU Merger Control System.

43. Pero también pueden incluirse las adquisiciones de Skype o Linkedln por Microsoft, de Waze por Google, de Kayak por Priceline. 
miembro pueden hacer a la Comisión. Y aún así, la operación fue aprobada sin condiciones en Fase 1.

Es claro que la Comisión subestimó en esa oportunidad la relevancia de la operación que tenía en sus manos, restando quizás importancia al hecho que el verdadero valor en juego era la capacidad de generar efectos de red para cerrar un mercado a terceros y acceder a datos de usuarios que hoy superan los mil millones en el mundo. Pine y Giangaspera ${ }^{44}$ nos recuerdan que en estos casos la competencia no se produce "en el mercado", sino "por el mercado"; esto es, por la posibilidad de ser el primero en lograr una posición hegemónica - first mover advantage- y alcanzar una envergadura tal que luego sea irreplicable por un competidor. Y el muy reciente anuncio de que Whatsapp empezaría a compartir formalmente los datos de sus usuarios con Facebook no es sino una confirmación de la importancia que tiene revisar a profundidad y oportunamente, con las obvias dificultades que supone, operaciones en estos mercados.

Esta situación, sin embargo, no es exclusiva de los mercados digitales, pudiendo presentarse en aquellos mercados en los que los costos de investigación y desarrollo son muy altos como por ejemplo, el sector farmacéutico y tecnológico, en general -45 , y en los que resulta particularmente rentable para ciertas empresas adquirir operaciones de negocios incipientes o aún en desarrollo con el fin de impedir su crecimiento y capturar mercados. A estas operaciones se les suele llamar killer acquisitions ${ }^{46}$ y explican por qué ciertos países han adoptado el criterio del valor de la transacción para determinar la obligacion de notificar. Alemania y Austria son los casos más recientes, incorpo- ADMINISTRATIVO rando en sus sistemas la obligación de notificar cuando el valor de la operación supere los 400 y 200 millones de Euros, respectivamente ${ }_{1}^{47}$ sumándose, entre otros, a Canadá, México y los Estados Unidos.

Si esto significa que las autoridades se volcarán unánimemente al uso del valor de transacción como un criterio de notificación está aún por verse. Por lo pronto, la Comisión Europea desestimó esta opción en el 2017, luego de una consulta efectuada con ese fin. ${ }^{48}$ No obstante ello, en la medida en que las herramientas convencionales sean insuficientes para enfrentar esta clase de problemas, es posible que se reconsidere recurrir a criterios como el valor de transacción, aunque presumiblemente siempre en combinación con otros criterios objetivos y de fácil determinación como las ventas o los ingresos de las partes involucradas.

\section{ALGUNAS REGLAS ESPECIALES PARA EL CÁLCULO DE UMBRALES}

Una vez definido el tipo de umbral aplicable — por ejemplo, ventas, ingresos brutos, valor

44. Chris Pike, y Matteo Giangaspero, Competition-for-the-Market (2019). OECD Competition Papers, disponible en: https://ssrn.com/abstract=3487669

45. Juliane Scholl, "Why the New Merger Control Thresholds in Germany?", Journal of European Competition Law \& Practices, Vol. 8, Number. 4, (2017)

46. Término acuñado para referirse a adquisiciones en la industria farmacéutica por Colleen Cunningham, Florian Ederer y Song Ma, "Killer Acquisitions" Journal of Political Economy, (April 19, 2020). disponible en: https://ssrn. com/abstract=3241707 o http://dx.doi.org/10.2139/ssrn.3241707.

Cunningham et al. estiman que el $6 \%$ de las adquisiciones en el sector —unas 45 por año-son killer acquisitions.

47. Bundeskartellamt, Guidance on transaction value thresholds for mandatory pre-merger notification (section 35 (1a) GWB and section 9 (4) KartG), 2018, https://www.bwb.gv.at/fileadmin/user_upload/Downloads/standpunkte/2018-07_Guidance_Transaction_Value_Thresholds.pdf

48. Comisión Europea, Consultation on evaluation of procedural and jurisdictional aspects of EU merger control, 2017, https://ec.europa.eu/competition/consultations/2016_merger_control/index_en.html 
de activos, entre otros-, cada legislación implementa una metodología específica para el cálculo de dichos valores, en virtud del tipo de acto de concentración de que se trate y, en ocasiones, considerando el mercado sobre el cual se ejecutará dicha operación.
En el caso del Perú, el artículo 4.2 del Reglamento de la Ley 31112 ha previsto las siguientes reglas de cálculo de umbrales, según el tipo de acto de concentración:

\section{Tipo de operación}

Fusiones, constitución de empresas en común, joint ventures o cualquier tipo de acuerdo colaborativo.

\section{Agente económico 1}

Valor de ventas o ingresos brutos anuales o el valor contable de los activos en el Perú de los agentes económicos participantes en la operación y de sus respectivos grupos económicos. ${ }^{49}$
Adquisición de derechos que permitan ejercer el control sobre la totalidad o parte de otro agente económico.
Valor de ventas o ingresos brutos anuales o el valor contable de los activos en el Perú del agente adquirente y de su grupo económico.
Valor de ventas o ingresos brutos anuales o el valor contable de los activos en el Perú del agente adquirido y de los agentes económicos sobre los cuales ejerce control.

Valor de ventas o ingresos brutos que hayan sido generados por los activos productivos operativos adquiridos o el valor contable de tales activos.
De esta manera, al igual que el tipo de umbral elegido, el Reglamento de la Ley 31112 ha seguido estándares internacionales para el cálculo de estos. ${ }^{50}$ En efecto, la exposición de motivos de dicho dispositivo ha indicado que, respecto de las operaciones de fusión, constitución de empresas en común, joint ventures o cualquier tipo de acuerdo asociativo, se ha tomado en consideración la regulación de la Dirección General de la Competencia de la Unión Europea y las agencias de competencia alemana - Bundeskartellamt-, española - Comisión Nacional de los Mercados y Competencia -CNMC - y chilena —Fiscalía Nacional Económica — FNE-.

Asimismo, en el caso de la adquisición de derechos que otorguen control, el Reglamento de la Ley 31112 ha recogido esta regla de la Dirección General de la Competencia de la Unión Europea, Bundeskartellamt, la CNMC y la FNE.

49. Cabe mencionar que tanto el Reglamento de la Ley 31112, como su exposición de motivos, establecen que cada empresa y su grupo económico constituyen un solo agente que interactúa en el mercado al ser controlados por la misma unidad de decisión.

50. Ver páginas de la 7 a la 10 de la exposición de motivos del Reglamento de la Ley 31112. 
Finalmente, en el caso de la adquisición de activos productivos operativos, la regla empleada es la utilizada por la FNE.

Sin perjuicio de que este desarrollo reglamentario realiza varias precisiones sobre el cálculo de umbrales - por ejemplo, saber cuándo se considerarán los ingresos brutos del grupo económico-, lo cierto es que aún existen algunos puntos por esclarecer. Por ello, no es coincidencia que la exposición de motivos del Reglamento de la Ley 31112 indique: “(...) a propuesta de la Secretaría Técnica, emitirá Lineamientos que describan de forma detallada los conceptos que deberán considerar las empresas para la determinación de su ventas o ingresos. ${ }^{151}$

En el caso concreto que atañe a esta sección expondremos, de un lado, (i) el tipo de umbrales que son aplicados por los países que forman parte de la Alianza Pacífico - entre ellos, Chile, Colombia y México-, cuya proximidad económica y geográfica, y su cercana colaboración el INDECOPI, hacen particularmente relevantes, así como en la Comisión Europea y los Estados Unidos de América, para luego analizar aquellos factores que son excluidos del cálculo de los umbrales; $y$, de otro, (ii) las reglas aplicables a casos especiales, en razón de su complejidad y/o del mercado que comprendería la operación. No obstante, cabe precisar que, dados los límites formales para la extensión del presente artículo, este no pretende ser un desarrollo exhaustivo, sino ofrecer un vistazo inicial sobre esta materia.

Dicho esto, a nivel de la Alianza Pacífico, se observa que Chile ha determinado sus umbrales en función de las ventas de los agentes económicos que participan del acto de concentración. Así, el artículo 48 del Decreto Ley 211 ha regulado los umbrales de la siguiente manera:

\section{"Deberán notificarse a la Fiscalía Nacional}

Económica, en forma previa a su perfeccionamiento, las operaciones de concentración que produzcan efectos en Chile y que cumplan con los siguientes requisitos copulativos:

a) Que la suma de las ventas en Chile de los agentes económicos que proyectan concen-ADMINISTRATIVO trarse haya alcanzado durante el ejercicio anterior a aquel en que se verifique la notificación, montos iguales o superiores al umbral establecido mediante resolución dictada por el Fiscal Nacional Económico.

b) Que, en Chile, por separado, al menos dos de los agentes económicos que proyectan concentrarse hayan generado ventas, $d u-$ rante el ejercicio anterior a aquel en que se verifique la notificación, por montos iguales o superiores al umbral establecido mediante resolución dictada por el Fiscal Nacional Económico". —Subrayado agregado-

De esta manera, pese a que el criterio de ventas empleado por su ley es amplio, en la práctica, la FNE ha entendido que no debe considerarse en el cálculo del umbral los impuestos directamente relacionados con el volumen de ventas - por ejemplo, el IGV o aranceles-, los montos descontados sobre el precio de venta - por ejemplo, descuentos por volumen-, las ventas generadas entre empresas del mismo grupo económico y toda aquella venta que no provenga de la explotación del giro habitual del negocio de las partes de la transacción o sus vinculadas. $^{52}$

En el caso de México, el artículo 86 de la Ley Federal de Competencia Económica, ha regulado los umbrales de la siguiente manera:

"Artículo 86. Las siguientes concentraciones deberán ser autorizadas por la Comisión antes de que se lleven a cabo:

51. Ver página 10 de la exposición de motivos del Reglamento de la Ley 31112.

52. Fiscalía Nacional Económica. "Guía práctica para la aplicación de umbrales de notificación de operaciones de concentración en Chile", (2017), acceso el 23 de enero de 2021, p. 5 y 6, https://www.fne.gob.cl/wp-content/ uploads/2017/10/Guia-Interpretacion-Umbrales-1.pdf 
1. Cuando el acto o sucesión de actos que les den origen, independientemente del lugar de su celebración, importen en el territorio nacional, directa o indirectamente, un monto superior al equivalente a dieciocho millones de veces el salario mínimo general diario vigente para el Distrito Federal;

II. Cuando el acto o sucesión de actos que les den origen, impliquen la acumulación del treinta y cinco por ciento o más de los activos o acciones de un Agente Económico, Cuyas ventas anuales originadas en el territorio nacional o activos en el territorio nacional importen más del equivalente a dieciocho millones de veces el salario mínimo general diario vigente para el Distrito Federal, o

III. Cuando el acto o sucesión de actos que les den origen impliquen una acumulación en el territorio nacional de activos o capital social superior al equivalente a ocho millones cuatrocientas mil veces el salario mínimo general diario vigente para el Distrito Federal y en la concentración participen dos o más Agentes Económicos cuyas ventas anuales originadas en el territorio nacional o activos en el territorio nacional conjunta o separadamente, importen más de cuarenta y ocho millones de veces el salario mínimo general diario vigente para el Distrito Federal."-Subrayado agregado-

Al igual que en el caso de Chile, pese a que la ley no haya precisado alguna excepción sobre la consideración de los activos en el cálculo de los umbrales, la agencia de competencia mexicana ha interpretado que no se debe tomar en cuenta aquellos activos que, pese a encontrar- se bajo el control de la empresa que es parte de la transacción, no son de su propiedad - por ejemplo, en los casos de empresas de maquila, las cesiones en uso de activos a efectos de que ejecuten el objeto del contrato-.$^{53}$

A nivel europeo, el artículo 1 del Reglamento -CE- No. 139/2004, Reglamento comunitario de concentraciones -en adelante, el "Reglamento de la $\mathrm{CE}^{\prime \prime}$ - precisa que los umbrales a nivel comunitario son los siguientes:

"2. Una concentración tendrá dimensión comunitaria cuando:

a) el volumen de negocios total a escala mundial realizado por el conjunto de las empresas afectadas supere los 5000 millones de euros, $y$

b) el volumen de negocios total a escala comunitaria realizado individualmente por al menos dos de las empresas afectadas por la concentración supere los 250 millones de euros, salvo que cada una de las empresas afectadas realice más de dos tercios de su volumen de negocios total comunitario en un mismo Estado miembro."-Subrayado agregado-

En este sentido, el artículo 5.1. del Reglamento de la $C E,{ }^{54}$ precisa que, a efectos del cálculo del volumen de ventas, no se toma en cuenta (i) los descuentos sobre ventas, (ii) los impuestos sobre el valor añadido u otros impuestos de naturaleza análoga y (iii) las transacciones generadas entre empresas de un mismo grupo económico.

53. COFECE, Guía para la notificación de concentraciones (2015) 20, https://www.cofece.mx/wp-content/ uploads/2017/12/guia-0042015_not_concentraciones.pdf

54. Reglamento (CE) №. 139/2004, Reglamento comunitario de concentraciones

"Artículo 5.- Cálculo del volumen de negocios

1. El volumen de negocios total en el sentido del presente Reglamento incluirá los importes resultantes de la venta de productos y la prestación de servicios por las empresas afectadas durante el último ejercicio correspondientes a sus actividades ordinarias, previa deducción de los descuentos sobre ventas, del impuesto sobre el valor añadido y de otros impuestos directamente relacionados con el volumen de negocios. El volumen de negocios total de una empresa afectada no tendrá en cuenta las transacciones que hayan tenido lugar entre las empresas contempladas en el apartado 4 del presente Artículo. (...)" 
Asimismo, la Comunicación consolidada de la Comisión sobre cuestiones jurisdiccionales en materia de competencia, realizada de conformidad con el Reglamento - CE- № 139/2004 del Consejo, sobre el control de las concentraciones entre empresas —-2008/C 95/01_ —en adelante, la "Comunicación de la CE" - precisa que, a efectos del cálculo del volumen de negocios, solo deberán tomarse en cuenta aquellos ingresos o ventas generadas en el marco de las "actividades ordinarias" de la empresa. ${ }^{55}$ Por dicha razón, como criterio general, serán excluidos aquellos registros contables que figuren en las partidas de "ingresos financieros" o "ingresos extraordinarios".

En el caso de los Estados Unidos de América, la Ley Hart-Scott-Rodino ha determinado como umbrales los siguientes criterios: ${ }^{56}$

(i) "Test del comercio: que alguna de las partes es un agente económico que participa en actividades comerciales o concurre en el mercado.

(ii) Test del tamaño de la transacción: que, como resultado del acto de concentración, el adquirente posea un importe agregado de acciones con derecho a voto, participaciones no societarias y/o activos de la parte adquirida superior a USD 94 millones.

(iii)Test del tamaño del agente: que el tamaño de la transacción supere los USD 94 millo- ADMINISTRATIVO nes, pero no los USD 367 millones, al menos una de las partes implicadas en la transacción debe tener unas ventas netas anuales o unos activos totales de al menos USD 188 millones y la otra debe tener unas ventas netas anuales o unos activos totales de al menos USD 18,8 millones."

Al igual que en los casos anteriores, las agencias de competencia de los Estados Unidos de América, han considerado que tampoco deberá tomarse en cuenta aquellos valores generados fuera del curso normal del negocio, tal como los registros contables anotados como "ingresos financieros" o "ingresos extraordinarios."

De esta manera, a modo de resumen, presentamos a continuación un cuadro que permite visualizar de manera integral los criterios antes expuestos:

55. Comunicación consolidada de la Comisión sobre cuestiones jurisdiccionales en materia de competencia, realizada de conformidad con el Reglamento (CE) No 139/2004 del Consejo, sobre el control de las concentraciones entre empresas (2008/C 95/01):

\section{“C. Dimensión Comunitaria \\ (...) \\ IV. Volumen de negocios \\ (...) \\ 2. Actividades ordinarias}

(161) El Artículo 5, apartado 1, dispone que los importes que han de tenerse en cuenta para calcular el volumen de negocios deberán corresponder a las «actividades ordinarias» de las empresas afectadas. Este volumen de negocios es el obtenido de la venta de productos o de la prestación de servicios en el normal desarrollo de la actividad. En general, excluye aquellas partidas que figuran en los epígrafes «ingresos financieros» 0 «ingresos extraordinarios» en las cuentas de la empresa. Tales ingresos extraordinarios pueden proceder de la venta de actividades o de activos fijos. Sin embargo, las cuentas de la empresa no delimitan necesariamente los ingresos resultantes de actividades ordinarias de la forma requerida a efectos de calcular el volumen de negocios con arreglo al Reglamento de concentraciones. En algunos casos, la denominación de las partidas en la contabilidad tal vez deba adaptarse a los requisitos del Reglamento de concentraciones."

56. Elizabeth Chen y James Lowe, USA: Merger control laws and regulations 2021, https://iclg.com/practice-areas/merger-control-laws-and-regulations/usa

57. Pedro Caro de Sousa, Local Nexus and jurisdictional thresholds in merger control. OCDE. Working party Number 3 on Co-operation and Enforcement. París: 2016. p. 10. Acceso el 25 de enero de 2021, https://www.oecd.org/ officialdocuments/publicdisplaydocumentpdf/?cote=DAF/COMP/WP3-2016)4\&docLanguage=En 
Factores excluidos por país

\begin{tabular}{l|l|l|l|l|l} 
& Chile & México & $\begin{array}{l}\text { Unión } \\
\text { Europea }\end{array}$ & EE.UU. & China $^{58}$ \\
$\begin{array}{l}\text { Impuestos directamente } \\
\text { relacionados con el volumen } \\
\text { de ventas. }\end{array}$ & Sí & - & Sí & - & Sí \\
\hline $\begin{array}{l}\text { Descuentos sobre las ventas. } \\
\text { Transacciones dentro del } \\
\text { mismo grupo económico. }\end{array}$ & Sí & - & Sí & - & Sí \\
\hline $\begin{array}{l}\text { Ingresos no correspondientes } \\
\text { al giro habitual de las partes o } \\
\text { sus vinculadas - por ejemplo, } \\
\text { ingresos financieros-. }\end{array}$ & Sí & - & Sí & Sí & - \\
\hline $\begin{array}{l}\text { Activos controlados pero } \\
\text { que no son propiedad de la } \\
\text { empresa. }\end{array}$ & - & Sí & - & - & - \\
\hline
\end{tabular}

De lo expuesto se puede concluir que la mayoría de estos criterios tienen en común como objeto que el monto final de las ventas refleje solo aquello que revele la verdadera aptitud de la empresa para competir en el mercado. Por ejemplo, las ventas generadas al interior de un mismo grupo económico no reflejan necesariamente la preferencia de la demanda sobre lo ofertado por esta empresa. Ello, pese a que la literalidad de sus respectivas normas de control de concentraciones no haya previsto una excepción o precisión a efectos del cálculo de sus umbrales.

Consideramos que estas interpretaciones sobre las respectivas normas de control de concentraciones son acertadas. Ello, en tanto que, de considerarse estos factores dentro del cálculo de los umbrales (i) representaría una dimen- sión no exacta de la relevancia competitiva de las partes del acto de concentración y (ii) en la práctica, motivaría que los umbrales objetivos pierdan su propósito principal: que las agencias de competencia analicen solo aquellas operaciones de concentración que revistan cierta relevancia competitiva.

Por todas estas razones, resultará indispensable que, en la emisión de sus lineamientos, el INDECOPI tome en cuenta la experiencia comparada para que los umbrales objetivos de la Ley 31112 no pierdan el sentido de su diseño y así evitar que, en los hechos, se notifiquen más operaciones de las que revisten una especial importancia en el mercado.

Finalmente, consideramos relevante enunciar brevemente cómo es que distintas jurisdiccio-

58. Pese a la juventud de la legislación de control de concentraciones de China, hemos considerado relevante mostrar los criterios asumidos por dicha jurisdicción, dada la mayor relevancia económica que dicha economía asiática ha tomado en los últimos años y la posible mayor actividad que tendrán los capitales de dicho país en las fusiones y adquisiciones con América Latina. 
nes han abordado algunos casos especiales, en razón de la complejidad del acto de concentración o del mercado en el que se desarrollaría el mismo:

\section{Fondos de inversión.}

En palabras de Víctor García-Vaquero, un fondo de inversión es un patrimonio colectivo que reúne aportaciones dinerarias de inversores individuales, cuya gestión en común se encomienda a un agente, al que se le suele denominar sociedad gestora del fondo de inversión..$^{59}$

De esta manera, en la medida en que buena parte de los fondos de inversión tienen participaciones en empresas de distintos mercados y países, resulta crucial adoptar un criterio que permita medir adecuadamente los umbrales en actos de concentración que involucren a dichos agentes. ${ }^{60}$

En el caso de Chile, a fin de calcular las ventas de un fondo de inversión, se toma en cuenta las comisiones asociadas al fondo y las ventas efectuadas por las empresas de su cartera, respecto de las cuales la sociedad gestora tenga control. ${ }^{61}$
Asimismo, en los actos de concentración que involucran una fusión o constitución conjunta de sociedades con un fondo de inversión, se deberá tomar en cuenta las ventas del grupo empresarial de la sociedad gestora. ${ }^{62}$ Esto también será aplicable cuando el fondo de inversión sea la parte adquirente en los casos de ad- ADMINISTRATIVO quisición de derechos que permitan el control o en la adquisición de activos.

En el caso europeo, la sección 5.3 de la Comunicación de la CE establece que se tomará en cuenta el volumen de negocios de toda la cartera de empresas detentada por los distintos fondos, respecto de las cuales se ejerza control. ${ }^{63}$

De igual forma, a nivel interno, se observa que España ha asumido un criterio similar, el cual se ha visto reflejado en el Informe N-04021 PPAC/ POLUPORE de la Dirección General de Defensa de la Competencia. ${ }^{64}$ En dicho caso, se analizó una operación en la que PP Acquisition Corporation - la cual era controlada por Warburg Pincus LLC - adquiriría el 100\% de acciones de Polypore Inc. De esta manera, como resultado de dicha adquisición, Polypore pasaría a ser

59. García-Vaquero, Víctor, Los fondos de inversión en España, (Lima: 1992) 5. Banco de España. Servicio de Estudios. Documento de trabajo Número 9202 https://www.bde.es/f/webbde/SES/Secciones/Publicaciones/PublicacionesSeriadas/DocumentosTrabajo/92/Fich/dt_9202.pdf

60. Así, a efectos de medir su representatividad en el mercado de fusiones y adquisiciones en el Perú, de la información publicada en Transactional Track Record (TTR), se observa que, desde enero de 2019 a enero de 2021, se han registrado 17 transacciones que han involucrado un fondo de inversión como por ejemplo, alcanzando una de estas operaciones un valor mayor a US\$ 280 millones.

61. Fiscalía Nacional Económica, Guía práctica para la aplicación de umbrales de notificación de operaciones de concentración en Chile (2017) 13. https://www.fne.gob.cl/wp-content/uploads/2017/10/Guia-Interpretacion-Umbrales-1. pdf

62. Cabe tener en cuenta que el grupo empresarial de la sociedad gestora incluye también a los otros fondos de inversión que esta administre.

63. Comunicación de la CE

"En consecuencia, esa organización de los distintos fondos por parte de la sociedad de inversión puede dar como resultado que el volumen de negocios de toda la cera de empresas detentada por los distintos fondos sea tenida en cuenta a efectos de evaluar si se alcanzan los umbrales del volumen de negocios del Artículo 1 si la sociedad de inversión adquiere el control indirecto de una cartera de empresas a través de alguno de los fondos".

64. Funciones que luego serían asumidas por la actual agencia de competencia española, la Comisión Nacional de los Mercados y la Competencia - CNMC. 
parte de los fondos de inversiones gestionados por Warburg Pincus LLC. ${ }^{65}$

Así, en dicho caso, la autoridad española tomó en cuenta el volumen de ventas de las empresas controladas por todos los fondos de inversión que gestionaba Warburg Pincus LLC.

En el caso de los Estados Unidos de América, la Ley de Mejoras Antimonopolio Hart-Scott-Rodino establece dos criterios para calcular los umbrales de los fondos de inversión. El primero corresponde al tamaño de la transacción, en escenarios de compra de acciones, derechos y/o activos represente un valor superior a un monto que es fijado anualmente por la autoridad, de acuerdo con la sección 16 CFR § 801.10. El segundo criterio corresponde al tamaño del agente que, en el caso de fondos de inversión, corresponde al Ultimate Parent Entity o la matriz que posee el control final efectivo. Para estos casos se toma en cuenta el valor neto de sus ventas y de sus activos.

\section{Empresas estatales.}

Cuando una empresa estatal forma parte de un acto de concentración, supone dificultades similares a las de los fondos de inversión, debido a su participación en diversas actividades económicas y, en algunos casos, en distintos niveles de la cadena.

Frente a ello, a efectos del cálculo de los umbrales, en Chile no se considera al Estado como una empresa o fondo de inversión, por lo que, a efectos de calcular las ventas del grupo económico de las empresas del Estado, únicamente se toma en cuenta aquellas que son controladas por este. ${ }^{66}$

En el caso europeo, la sección 5.4 del capítulo IV de la Comunicación de la CE precisa que "a los efectos de calcular el volumen de negocios de empresas de propiedad estatal, sólo se tendrá en cuenta a aquellas empresas que pertenezcan a la misma entidad económica y tengan el mismo poder de decisión autónomo". Es decir, se tomará en cuenta solo aquellas empresas respecto de las cuales el Estado tenga control -y, por consiguiente, relación directa con la gestión-y no solo una simple participación accionaria.

\section{Comercio exterior.}

El caso de actividades de comercio exterior importaciones y/o exportaciones- supone cierta complejidad a efectos de determinar qué parte del volumen de negocio cumple con el requisito de nexo geográfico de la autoridad que va a analizar el acto de concentración. En este rubro, las autoridades no han mostrado una perspectiva unánime respecto de, por ejemplo, ¿qué ventas considerar en el caso de una empresa exportadora? ¿Una concentración en dicho mercado cumple con el criterio de nexo geográfico al tener incidencia directa en el país?

En el caso de Colombia, se ha indicado que, cuando una de las partes participe en el mercado exclusivamente a través de exportaciones hacia Colombia, y no tenga ingresos, operaciones o activos totales en dicho país, se contabilizarán los activos totales e ingresos de la empresa en el extranjero y los de aquellas que (i) se encuentren vinculadas en virtud del

65. Ver pie de página 1 del referido informe.

66. Guía práctica para la aplicación de umbrales de notificación de operaciones de concentración en Chile: "IX. Disposiciones especiales para empresas del Estado

40. Tratándose de empresas públicas, es necesario tener algunas precauciones al momento de determinar las ventas del grupo empresarial. En concreto, no debe considerarse al Estado como una empresa o sociedad de inversiones, por lo que para calcular las ventas del grupo empresarial de entidades de propiedad estatal sólo se toma en cuenta a aquellas empresas que pertenezcan al mismo centro independiente de toma de decisiones. Es decir, no se considera como parte del grupo empresarial a sociedades relacionadas indirectamente a través del Estado, pero sin ninguna relación en términos de administración." 
control que ejerza sobre ellas dicha empresa y (ii) desarrollen la misma actividad económica o se encuentren dentro de la misma cadena productiva de las partes del acto de concentración. ${ }^{67}$

En contraste, en Chile el criterio se ha enfocado en la ubicación geográfica; esto es, el lugar donde se ejecute la prestación final. Así, por ejemplo, en los casos en que la obligación principal sea el abastecer productos o prestar servicios localizados fuera del país, no se computarán las ventas asociadas a dichas actividades. De esta manera, aunque un contrato sea celebrado en el extranjero, si es que la ejecución de la prestación se produce dentro del país, las ventas de dicha actividad sí se tomarán en cuenta a efectos del cálculo de los umbrales. Así, el lugar de la ejecución de la prestación principal será el elemento determinante, por encima de (i) la ubicación del cliente al momento de celebrar el contrato o (ii) la ubicación de la sociedad a la cual se le facture el servicio. ${ }^{68}$

Asimismo, la "Guía práctica para la aplicación de umbrales de notificación de operaciones de concentración en Chile" ha precisado que, en el caso que una compañía multinacional tuviese una unidad de compras constituida en otro país, que se encargase de centralizar el abastecimiento de la matriz, para luego redistribuir dichos productos a puntos de venta en distintos países, se tomará en cuenta úni- camente las ventas correspondientes al país donde se centralizaron las compras de dichos productos. ${ }^{69}$

Finalmente, a nivel europeo se toma en cuenta el lugar donde se preste efectivamente el servicio o se entregue el producto. Así, a modo de ejemplo, el Comunicado de la CE indica que, si un producto es vendido vía telefónica o por internet y el proveedor se encuentra en otro país, lo determinante será el lugar en donde se entrega finalmente el bien. En los términos del artículo 5 del Comunicado de la $C E$, la lógica que subyace a dicho criterio es que el volumen de negocios calculado esté vinculado con el "lugar donde se ha producido la competencia con otros posibles proveedores".

Así pues, dicho dispositivo establece que, en aquel caso en que una matriz tuviese filiales en distintos países y que, estas últimas fuesen las unidades demandantes de productos en dichos países, pese a que la matriz fuese la sociedad que pagase por estos, se tomará en cuenta únicamente las ventas del país en el que esté ubicada dicha filial. Tal y como en el caso anterior, la lógica subyacente es que el nexo geográfico se encuentra vinculado al lugar en donde se produce la competencia por el producto. Ello, en la medida que son las propias filiales los agentes que deciden contratar $y$, en consecuencia, ejercen influencia directa sobre la demanda en dicho mercado.

67. Al respecto, ver Artículo 2.1.2 de la Resolución № 10930 de 2015, por la cual se modifica el Capítulo Segundo del Título VII de la Circular Única de la Superintendencia de Industria y Comercio. Acceso el 27 de enero de 2021. https://www.sic.gov.co/sites/default/files/files/Resoluci\%C3\%B3n\%2010930\%20de\%202015(1).pdf

68. Guía práctica para la aplicación de umbrales de notificación de operaciones de concentración en Chile "V. Asignación geográfica del volumen de ventas

(...)

Como norma general, la Fiscalía considera apropiada la asignación de las ventas de acuerdo a la localización de los clientes al momento en que se celebra el contrato de compraventa, sin importar el país donde se haya celebrado éste. Esto coincide normalmente con el lugar en que se presta el servicio o se provee finalmente el producto. De este modo, contratos celebrados en Chile con el objeto final de abastecer de productos o proveer servicios a clientes localizados fuera del país, no deben ser contabilizados. Por el contrario, aquellos contratos celebrados en el extranjero con el propósito final de abastecer productos o prestar servicios a clientes localizados en Chile, sí deben ser consideradas para determinar el traspaso de los umbrales definidos por la Fiscalía."

69. Ver párrafo 24 de la referida guía. 
En el caso de servicios, la Comunicación de la CE subdivide la casuística en (i) aquella en la que se desplaza el proveedor, (ii) aquella en la que se desplaza el cliente y (iii) aquella en la que no se requiere el desplace del proveedor ni del cliente. En los dos primeros casos, se imputa el volumen de negocios del lugar de destino de quien se desplaza y, en el tercer caso, se toma en cuenta el volumen de negocio del lugar en donde se encuentre el cliente, esto es, la fuerza de la demanda.

\section{NOTA FINAL}

El presente trabajo busca examinar, desde su teoría y práctica, uno de los elementos centrales para la aplicación del nuevo marco legal sobre control de concentraciones empresariales en nuestro país; esto es, los umbrales de notificación. Hemos tratado de ofrecer una visión panorámica sobre esta materia y de fijar los ejes centrales para una aproximación académica, pero también práctica. Es evidente que lo desarrollado en estas páginas no agota la discusión sobre los umbrales y su aplicación, y está bien que así sea. Esta es una historia que recién empieza y se espera que este trabajo sirva como catalizador para una mayor discusión sobre este tema. 